\title{
Existence of Solutions for Critical Systems with Variable Exponents
}

\section{Hadjira Lalili ${ }^{a}$, Saâdia Tas $^{a}$ and Ali Djellit ${ }^{b}$}

${ }^{a}$ Applied Mathematics Laboratory, Faculty of Exact Sciences, University of Béjaïa

Béjaïa 06000, Algeria

${ }^{b}$ Mathematics, Dynamics and Modelization Laboratory, University of Annaba Annaba 23000, Algeria

E-mail(corresp.): h_lalili@yahoo.com

E-mail: tas_saadia@yahoo.fr

E-mail: a_djellit@hotmail.com

Received September 22, 2017; revised September 8, 2018; accepted September 9, 2018

Abstract. In this work, we deal with elliptic systems under critical growth conditions on the nonlinearities. Using a variant of concentration-compactness principle, we prove an existence result.

Keywords: $\mathrm{p}(\mathrm{x})$-Laplacian, generalized Sobolev spaces, critical Sobolev exponents, concentration-compactness principle, critical points theory.

AMS Subject Classification: 35B33; 35J50; 35J60.

\section{Introduction}

Let $\Omega$ be a bounded domain in $\mathbb{R}^{N}(N \geq 2)$ with smooth boundary $\partial \Omega$. We consider the following nonlinear elliptic systems of the form

$$
\left\{\begin{array}{l}
-\Delta_{p(x)} u=|u|^{\alpha(x)-2} u+\lambda \frac{\partial F}{\partial u}(x, u, v), \quad \text { in } \Omega, \\
-\Delta_{q(x)} v=|v|^{\beta(x)-2} v+\lambda \frac{\partial F}{\partial v}(x, u, v), \quad \text { in } \Omega, \\
u=0, \quad v=0, \quad \text { on } \partial \Omega,
\end{array}\right.
$$

where $\lambda$ is a positive parameter and $\alpha, \beta, p, q: \bar{\Omega} \rightarrow \mathbb{R}$ are Lipschitz continuous functions verifying

$$
1<p^{-}:=\inf _{\bar{\Omega}} p(x) \leq p^{+}:=\sup _{\bar{\Omega}} p(x)<N, \quad 1<q^{-}:=\inf _{\bar{\Omega}} q(x) \leq q^{+}:=\overline{s u p}_{\bar{\Omega}} q(x)<N,
$$




$$
\begin{aligned}
& 1 \leq \alpha(x) \leq p^{*}(x)=\frac{N p(x)}{N-p(x)}, \quad 1 \leq \beta(x) \leq q^{*}(x)=\frac{N q(x)}{N-q(x)}, \quad \forall x \in \Omega, \\
& A_{p}=\left\{x \in \Omega, \quad \alpha(x)=p^{*}(x)\right\} \neq \emptyset, \quad A_{q}=\left\{x \in \Omega, \quad \beta(x)=q^{*}(x)\right\} \neq \emptyset .
\end{aligned}
$$

We designate by $\left(\frac{\partial F}{\partial u}, \frac{\partial F}{\partial v}\right)$ the gradient of the potential $F: \bar{\Omega} \times \mathbb{R}^{2} \rightarrow \mathbb{R}$ and $\Delta_{r(.)} u:=\operatorname{div}\left(|\nabla u|^{r(.)-2} \nabla u\right)$ is the so-called $r($.$) -Laplacian operator.$

Recently, elliptic equations involving variable exponents with non-standard growth conditions have been developed very markedly in last decade due to the fact that they have arisen in the mathematical modeling of various physical processes, as in nonlinear elasticity theory, electrorheological fluids dynamics and image restorations. Such problems have attracted an increasing attention and many results have been obtained by several authors. We would mention to ( [3], [4], [16], [19], [20], [26], [30], [31]) and survey papers ( [8], [10], [14], [21], $[24],[27],[29])$ for the advances and references in this area.

In particular, it's well known that the class of quasilinear elliptic problems with constant critical exponents in bounded or unbounded domain occupies a considerable place in the literature, which was discussed for the first time in the seminal paper [7] by Brezis-Nirenberg. Afterward, Lions [25] established the concentration-compactness principle in the limit case in the calculus of variation and it became one of the main techniques played an important role in order to deal with such issues. Several results have been obtained by variational methods, thus, it would be interesting to refer the reader to some works for gradient type in the constant exponent case.

Djellit and Tas [11] established the existence of nontrivial weak solutions for the systems

$$
\begin{cases}-\Delta_{p} u=f(x)|u|^{p^{*}-2} u+\lambda F_{u}(x, u, v), & \text { in } \mathbb{R}^{N} \\ -\Delta_{q} v=g(x)|v|^{q^{*}-2} v+\lambda F_{v}(x, u, v), & \text { in } \mathbb{R}^{N} \\ u, v \rightarrow 0, \text { as }|x| \rightarrow \infty & \end{cases}
$$

for all $\lambda \in\left(0, \lambda_{1}\right)$ by using Lions's principe with mountain pass theorem. Here $\lambda_{1}$ is the first eigenvalue of the system

$$
\left\{\begin{array}{l}
-\Delta_{p} u=\lambda F_{u}(x, u, v), \quad \text { in } \mathbb{R}^{N}, \\
-\Delta_{q} v=\lambda F_{v}(x, u, v), \quad \text { in } \mathbb{R}^{N} \\
u, v \rightarrow 0, \quad \text { as }|x| \rightarrow \infty, u>0, \quad v>0
\end{array}\right.
$$

Martínez and Rossi [6] inspected in detail the following system on bounded set of $\mathbb{R}^{N}$ with nonlinear coupling at the boundary

$$
\left\{\begin{array}{l}
-\Delta_{p} u=|u|^{p-2} u \\
-\Delta_{q} v=|v|^{q-2} v \\
|u|^{p-2} u \frac{\partial u}{\partial \nu}=F_{u}(x, u, v),|v|^{q-2} v \frac{\partial v}{\partial \nu}=F_{v}(x, u, v) .
\end{array}\right.
$$

They proved the existence of positive solutions under suitable assumptions on the potential $F$ based on variational arguments. The authors examined the subcritical, resonant and critical growth on $F$. Knowing that this contribution comes within the generalization results described on [1] and [2]. 
In a natural way, Lions's principle is generalized to the variable exponents setting by Bonder and Silva in [5], and they proved an existence result to the following equation

$$
-\Delta_{p(x)} u=|u|^{q(x)-2} u+\lambda(x)|u|^{r(x)-2} u, \quad \text { in } \Omega,
$$

where $\left\{x \in \Omega, \quad q(x)=p^{*}(x)\right\} \neq \emptyset, \Omega$ is a bounded smooth domain in $\mathbb{R}^{N}$ and $r(x)<p(x)-\delta, q(x) \leq p^{*}(x)$. Moreover, Silva in [28], considered the following critical equation

$$
-\Delta_{p(x)} u=|u|^{q(x)-2} u+\lambda f(x, u), \quad \text { in } \Omega,
$$

with $\left\{x \in \Omega, \quad q(x)=p^{*}(x)\right\} \neq \emptyset$. Applying variational method with the above principle, the author proved the existence of three nontrivial solutions.

On the other hand, Fu in [18] established comparable results on the concentration-compactness principle in $W^{1, p(x)}(\Omega)$ and discuss existence results for $p(x)$-Laplacian equation involving critical exponents

$$
-\Delta_{p(x)} u+\lambda|u|^{q(x)-2} u=f(x, u)+h(x)|u|^{p^{*}(x)-2} u, \quad \text { in } \mathbb{R}^{N},
$$

Shortly afterward, Zhang and Fu modify the principle of concentrationcompactness in $W^{1, p(x)}\left(\mathbb{R}^{N}\right)$ by proving typical Sobolev inequalities (see [32]). Then, by using variational method, they obtained the existence of weak solutions for the following critical equation

$$
-\Delta_{p(x)} u+|u|^{q(x)-2} u=|u|^{p^{*}(x)-2} u+h(x), \quad \text { in } \mathbb{R}^{N},
$$

when the perturbation is small enough.

Our objective in this article is to study the existence of nontrivial weak solutions for system (1.1). Precisely, following the same ideas introduced in [2], [6] and [11], we overcome the difficulties caused by the lack of compactness in Sobolev embedding using a variant of concentration-compactness principle on variable exponents Sobolev spaces and mountain pass theorem.

The rest of this paper is organized as follows: in Section 2, we recall some definitions and basic properties on variable exponents spaces. Section 3 is devoted to the proof of the main results.

\section{Preliminary results and hypotheses}

It's well-known that the generalized Lebesgue-Sobolev spaces practically enjoy the same Sobolev spaces properties. Unfortunately, convolution and translation properties doesn't hold, (see [9], [13], [15], [17]).

Set

$$
C_{+}(\bar{\Omega})=\{h \in C(\bar{\Omega}) ; h(x)>1, \quad \forall x \in \bar{\Omega}\} .
$$

For $p \in C_{+}(\bar{\Omega})$, let $L^{p(x)}(\Omega)$ be the collection of all functions $u: \Omega \subset \mathbb{R}^{N} \rightarrow \mathbb{R}$, such that

$$
\varrho(u)=\int_{\Omega}|u(x)|^{p(x)} d x<+\infty,
$$


equipped with the Luxemburg norm

$$
\|u\|_{p(x)}:=|u|_{L^{p(x)}(\Omega)}=\inf \{\lambda>0: \varrho(u(x) / \lambda)<1\} .
$$

We denote by $L^{p^{\prime}(x)}(\Omega)$ the Lebesgue dual space, i.e. $p^{\prime}(x)=\frac{p(x)}{p(x)-1}$. The variable exponent Sobolev space $W^{1, p(x)}(\Omega)$ is defined by

$$
W^{1, p(x)}(\Omega)=\left\{u: \Omega \subset \mathbb{R}^{N} \rightarrow \mathbb{R}: u \in L^{p(x)}(\Omega),|\nabla u| \in L^{p(x)}(\Omega)\right\},
$$

endowed with the usual norm

$$
\|u\|_{1, p(x)}=\|u\|_{p(x)}+\|\nabla u\|_{p(x)} .
$$

The space $W_{0}^{1, p(x)}(\Omega)$ is defined as the closure of $C_{0}^{\infty}(\Omega)$ with respect to the norm $\|u\|_{1, p(x)}$. In this way, $L^{p(x)}(\Omega), W^{1, p(x)}(\Omega)$ and $W_{0}^{1, p(x)}(\Omega)$ are separable and reflexive Banach spaces ( $[13],[17])$.

Proposition 1. ([13], [17]) For $u \in L^{p(x)}(\Omega)$ and $v \in L^{p^{\prime}(x)}(\Omega)$, we have the Hölder's inequality

$$
\int_{\Omega}|u v| d x \leq\left(\frac{1}{p^{-}}+\frac{1}{p^{\prime}-}\right)|u|_{p(x)}|v|_{p^{\prime}(x)} \leq 2|u|_{p(x)}|v|_{p^{\prime}(x)} .
$$

Proposition 2. ([13], [17]) If $u \in L^{p(x)}(\Omega)$, then

$$
\min \left\{|u|_{p(x)}^{p^{-}},|u|_{p(x)}^{p^{+}}\right\} \leq \varrho(u) \leq \max \left\{|u|_{p(x)}^{p^{-}},|u|_{p(x)}^{p^{+}}\right\}
$$

In addition, we have

1. $|u|_{p(x)}<1($ resp. $=1,>1) \Leftrightarrow \varrho(u)<1($ resp. $=1,>1)$,

2. $|u|_{p(x)}<1 \Rightarrow|u|_{p(x)}^{p^{+}} \leq \varrho(u) \leq|u|_{p(x)}^{p^{-}}$,

3. $|u|_{p(x)}>1 \Rightarrow|u|_{p(x)}^{p^{-}} \leq \varrho(u) \leq|u|_{p(x)}^{p^{+}}$,

4. $\varrho\left(u /|u|_{p(x)}\right)=1$.

Proposition 3. ([13], [15]) Let $p(x)$ and $q(x)$ be measurable functions such that $p(x) \in L^{\infty}(\Omega)$ and $1 \leq p(x) q(x) \leq \infty$ a.e. in $\Omega$. If $u \in L^{q(x)}(\Omega), u \neq 0$. Then

$$
\begin{gathered}
|u|_{p(x) q(x)} \leq 1 \Rightarrow|u|_{p(x) q(x)}^{p^{-}} \leq \|\left.\left. u\right|^{p(x)}\right|_{q(x)} \leq|u|_{p(x) q(x)}^{p^{+}} \\
|u|_{p(x) q(x)} \geq 1 \Rightarrow|u|_{p(x) q(x)}^{p^{+}} \leq\left.\left.|| u\right|^{p(x)}\right|_{q(x)} \leq|u|_{p(x) q(x)}^{p^{-}}
\end{gathered}
$$

In particular, if $p(x)=p$ is a constant, then $\|\left.\left. u\right|^{p}\right|_{q(x)}=|u|_{p q(x)}^{p}$.

Proposition 4. ([13], [15]) If $u, u_{n} \in L^{p(x)}(\Omega), n=1,2, \ldots$, then the following statements are mutually equivalent 
1. $\lim _{n \rightarrow \infty}\left|u_{n}-u\right|_{p(x)}=0$,

2. $\lim _{n \rightarrow \infty} \varrho\left(u_{n}-u\right)=0$,

3. $u_{n} \rightarrow u$ in measure in $\Omega$ and $\lim _{n \rightarrow \infty} \varrho\left(u_{n}\right)=\varrho(u)$.

Proposition 5. ([13], [15]) If $p(x): \bar{\Omega} \rightarrow \mathbb{R}$ is Lipschitz-continuous function, then there exists a positive constant $c$ such that

$$
\|u\|_{p^{*}(x)} \leq c\|u\|_{1, p(x)}, \quad \forall u \in W_{0}^{1, p(x)}(\Omega) .
$$

Proposition 6. ( [9], [17]) Let $p, q \in C(\bar{\Omega})$ be such that $1 \leq q(x) \leq p^{*}(x)$ for all $x \in \bar{\Omega}$. Assume moreover that the function $p$ and $q$ are log-Hölder continuous. Then the embedding $W^{1, p(x)}(\Omega) \hookrightarrow L^{q(x)}(\Omega)$ is continuous. Moreover, if $\inf \left(p^{*}-q\right)>0$ then, the embedding $W^{1, p(x)}(\Omega) \hookrightarrow L^{q(x)}(\Omega)$ is compact. $\Omega$

Proposition 7. ([9], [17]) There is a constant $C>0$ such that

$$
\|u\|_{p(x)} \leq C\|\nabla u\|_{p(x)}, \quad \text { for all } u \in W_{0}^{1, p(x)}(\Omega) .
$$

$\|u\|_{p(x)}=\|\nabla u\|_{p(x)}$ and $\|u\|_{1, p(x)}$ are equivalent norms on $W_{0}^{1, p(x)}(\Omega)$.

The main tool used to prove the existence results is mountain pass theorem. Nevertheless, the lack of compactness in critical generalized Sobolev embedding leads to convergence problem. Fortunately unbounded Section 3, the technical used here is the following principle of concentration-compactness in $W^{1, p(x)}(\Omega)$ established in [5].

Proposition 8. Let $p(x)$ and $q(x)$ be two Lipschitz continuous functions such that

$$
1<\inf _{\bar{\Omega}} p \leq \sup _{\bar{\Omega}} p<N \quad \text { and } \quad 1 \leq q(x) \leq p^{*}(x) \quad \text { in } \Omega .
$$

Let $\left\{u_{n}\right\}_{n \in \mathbb{N}}$ be a weakly convergent sequence in $W_{0}^{1, p(x)}(\Omega)$ with weak limit $u$, and such that

$$
\begin{aligned}
& \left|\nabla u_{n}\right|^{p(x)} \rightarrow \mu \quad \text { (weakly in sense of measures) }, \\
& \left|u_{n}\right|^{q(x)} \rightarrow \nu \quad \text { (weakly in sense of measures). }
\end{aligned}
$$

Assume moreover that $A_{p}=\left\{x \in \Omega, q(x)=p^{*}(x)\right\}$ is nonempty.Then for some countable index set $J$, we have

$$
\begin{aligned}
& \nu=|u|^{q(x)}+\sum_{j \in J} \nu_{j} \delta_{x_{j}}, \quad \nu_{j}>0, \quad \mu \geq|\nabla u|^{p(x)}+\sum_{j \in J} \mu_{j} \delta_{x_{j}}, \quad \mu_{j}>0, \\
& S_{p} \nu_{j}^{\frac{1}{p^{*}\left(x_{j}\right)}} \leq \mu_{j}^{\frac{1}{p\left(x_{j}\right)}}, \quad \forall j \in J .
\end{aligned}
$$

Here $\delta_{x_{j}}$ is the Dirac measure at $\left.x_{j},\left\{x_{j}\right\} \subset A_{p},\left\{\mu_{j}\right\},\left\{\nu_{j}\right\} \subset\right] 0,+\infty\left[\right.$ and $S_{p}$ is the best constant in the Gagliardo-Nirenberg-Sobolev inequality for variable exponents corresponding to the embedding $W^{1, p(x)}(\Omega) \hookrightarrow L^{q(x)}(\Omega)$, namely

$$
S_{p}=S(p(x), q(x), \Omega)=\inf _{u \in W_{0}^{p(x)}(\Omega)} \frac{\|\nabla u\|_{p(x)}}{\|u\|_{q(x)}}, u \neq 0 .
$$


We denote $X$ the product space $W_{0}^{1, p(x)}(\Omega) \times W_{0}^{1, q(x)}(\Omega)$ endowed with the norm

$$
\|(u, v)\|_{p(x), q(x)}=\max \left\{\|\nabla u\|_{p(x)},\|\nabla v\|_{q(x)}\right\} .
$$

For every $(u, v)$ in $X$, let

$$
\begin{aligned}
J(u, v) & =\int_{\Omega} \frac{1}{p(x)}|\nabla u|^{p(x)} d x+\int_{\Omega} \frac{1}{q(x)}|\nabla v|^{q(x)} d x, \\
K(u, v) & =\int_{\Omega} F(x, u(x), v(x)) d x \\
L(u, v) & =\int_{\Omega} \frac{1}{\alpha(x)}|u|^{\alpha(x)} d x+\int_{\Omega} \frac{1}{\beta(x)}|v|^{\beta(x)} d x, \\
I_{\lambda}(u, v) & =J(u, v)-L(u, v)-\lambda K(u, v), \forall \lambda>0 .
\end{aligned}
$$

In order to guarantee that the functional $I_{\lambda}$ satisfies the topological hypothesis and the geometric assumptions of mountain pass theorem (see [22] or [23]), we suppose that

$\left(\mathcal{H}_{1}\right)$ The nonlinearity $F \in C^{1}\left(\mathbb{R}^{N} \times \mathbb{R}^{2}, \mathbb{R}^{+}\right)$and satisfied $F(x, 0,0)=0$.

$\left(\mathcal{H}_{2}\right)$ There exists positive functions $a_{i}, b_{i},(i=1,2)$ such that

$$
\begin{aligned}
& \left|\frac{\partial F}{\partial u}(x, u, v)\right| \leq a_{1}(x)|u|^{p_{1}^{-}-1}+a_{2}(x)|v|^{p_{1}^{+}-1}, \\
& \left|\frac{\partial F}{\partial v}(x, u, v)\right| \leq b_{1}(x)|u|^{q_{1}^{-}-1}+b_{2}(x)|v|^{q_{1}^{+}-1},
\end{aligned}
$$

where $1<p_{1}(x), q_{1}(x) \leq \inf (p(x), q(x))$, for all $x \in \Omega$. The weight functions $a_{i}$ and $b_{i},(i=1,2)$ belong respectively to the generalized Lebesgue spaces $L^{\alpha_{i}(x)}(\Omega)$ and $L^{\sigma(x)}(\Omega)$, where

$$
\alpha_{1}(x)=\frac{p(x)}{p(x)-1}, \quad \sigma(x)=\frac{p^{*}(x) q^{*}(x)}{p^{*}(x) q^{*}(x)-p^{*}(x)-q^{*}(x)}, \quad \alpha_{2}(x)=\frac{q(x)}{q(x)-1} .
$$

$\left(\mathcal{H}_{3}\right) \exists M>0, \exists \theta_{1} \in\left(p^{+}, \alpha^{-}\right), \exists \theta_{2} \in\left(q^{+}, \beta^{-}\right)$, for all $(x, u, v) \in\left(\Omega, \mathbb{R}^{2}\right)$ where $|u|^{\theta_{1}},|v|^{\theta_{2}} \geq M$

$$
0<F(x, u, v) \leq \frac{u}{\theta_{1}} \frac{\partial F}{\partial u}(x, u, v)+\frac{v}{\theta_{2}} \frac{\partial F}{\partial v}(x, u, v) .
$$

$\left(\mathcal{H}_{4}\right) \exists c>0$ such that

$$
|F(x, u, v)| \leq c\left(|u|^{r_{1}(x)}+|v|^{r_{2}(x)}\right), \quad \forall(x, u, v) \in\left(\Omega, \mathbb{R}^{2}\right),
$$

where $r_{1}, r_{2} \in C_{+}\left(\bar{\Omega}, p^{+}<r_{1}^{-} \leq r_{1}^{+} \ll \alpha^{-} \leq \alpha^{+}\right.$and $q^{+}<r_{2}^{-} \leq r_{2}^{+} \ll \beta^{-} \leq \beta^{+}$.

An example of such functions is $F(x, u, v)=a(x)|u|^{\gamma(x)}|v|^{\delta(x)}$, where $\gamma(x)$, $\delta(x) \geq 1$ and $a \in L^{\theta(x)}(\Omega) \cap L^{\infty}(\Omega)$ is positive with

$$
\theta(x)=\frac{p^{*}(x) q^{*}(x)}{p^{*}(x) q^{*}(x)-\gamma(x) q^{*}(x)-\delta(x) p^{*}(x)}, \frac{\gamma(x)}{p(x)}+\frac{\delta(x)}{q(x)}>1, \frac{\gamma(x)}{p^{*}(x)}+\frac{\delta(x)}{q^{*}(x)}<1 .
$$

It is easy to check that $\left(\mathcal{H}_{1}\right)$ holds, Young's inequality gives $\left(\mathcal{H}_{2}\right)$ and $\left(\mathcal{H}_{4}\right)$. Moreover; $\left(\mathcal{H}_{3}\right)$ holds if the functions $\gamma$ and $\delta$ are such that $\frac{\gamma^{-}}{\theta_{1}}+\frac{\delta^{-}}{\theta_{2}} \geq 1$. 
Notice that under the above assumptions $I_{\lambda}$ is well defined and is of class $C^{1}(X, \mathbb{R})$. Moreover, for all $(u, v),(\varphi, \psi) \in X$

$$
\begin{aligned}
I_{\lambda}^{\prime}(u, v)(\varphi, \psi)= & \int_{\Omega}|\nabla u|^{p(x)-2} \nabla u \nabla \varphi d x+\int_{\Omega}|\nabla v|^{q(x)-2} \nabla v \nabla \psi d x \\
& -\int_{\Omega}|u|^{\alpha(x)-2} u \varphi d x-\int_{\Omega}|v|^{\beta(x)-2} v \psi d x \\
& -\lambda \int_{\Omega} \frac{\partial F}{\partial u}(x, u, v) \varphi d x-\lambda \int_{\Omega} \frac{\partial F}{\partial v}(x, u, v) \psi d x, \forall \lambda>0 .
\end{aligned}
$$

The dual space $X$ is denoted by $X^{*}$ and $\|\cdot\|_{*}$ stands its norm. Hence, weak solutions of the system (1.1) are exactly critical points of the functional $I_{\lambda}$.

Now, we can state the following existence result.

Theorem 1. If the hypotheses $\left(\mathcal{H}_{1}\right)-\left(\mathcal{H}_{4}\right)$ are satisfied, then there exists a constant $\bar{\lambda}>0$ such that, if $\lambda>\bar{\lambda}$, the problem (1.1) has at least one weak solution in $X$.

\section{Main results}

We need some lemmas to prove the main theorem.

Lemma 1. Under the assumptions $\left(\mathcal{H}_{1}\right)$ and $\left(\mathcal{H}_{2}\right)$, the functional $K$ is well defined, lower weakly semicontinuous and it is of class $C^{1}$ in $X$. Moreover, the operator $K^{\prime}$ is compact from $X$ to $X^{*}$.

The proof of the above Lemma follows the same arguments as in [12].

Lemma 2. Let $\left(u_{n}, v_{n}\right) \subset X$ be a Palais-Smale sequence for the Euler-Lagrange functional $I_{\lambda}$, then under hypothesis $\left(\mathcal{H}_{3}\right),\left(u_{n}, v_{n}\right)$ is bounded.

Proof. Let $\left(u_{n}, v_{n}\right) \subset X$ be a Palais-Smale sequence, we have

$$
\begin{aligned}
& I_{\lambda}\left(u_{n}, v_{n}\right)=\int_{\Omega}\left[\frac{\left|\nabla u_{n}\right|^{p(x)}}{p(x)}-\frac{\left|u_{n}\right|^{\alpha(x)}}{\alpha(x)}+\frac{\left|\nabla v_{n}\right|^{q(x)}}{q(x)}-\frac{\left|v_{n}\right|^{\beta(x)}}{\beta(x)}\right. \\
& \left.-\lambda F\left(x, u_{n}, v_{n}\right)\right] d x=C+o(1) .
\end{aligned}
$$

On the other hand

$$
\begin{aligned}
& I_{\lambda}^{\prime}\left(u_{n}, v_{n}\right)(\varphi, \psi)=\int_{\Omega}\left|\nabla u_{n}\right|^{p(x)-2} \nabla u_{n} \nabla \varphi d x+\int_{\Omega}\left|\nabla v_{n}\right|^{q(x)-2} \nabla v_{n} \nabla \psi d x \\
& \quad-\int_{\Omega}\left|u_{n}\right|^{\alpha(x)-2} u_{n} \varphi d x-\int_{\Omega}\left|v_{n}\right|^{\beta(x)-2} v_{n} \psi d x \\
& \quad-\lambda \int_{\Omega} \frac{\partial F}{\partial u}\left(x, u_{n}, v_{n}\right) \varphi d x-\lambda \int_{\Omega} \frac{\partial F}{\partial v}\left(x, u_{n}, v_{n}\right) \psi d x=o(1) .
\end{aligned}
$$


Then

$$
\begin{aligned}
I_{\lambda}\left(u_{n}, v_{n}\right)-I_{\lambda}^{\prime}\left(u_{n}, v_{n}\right)\left(\frac{u_{n}}{\theta_{1}}, \frac{v_{n}}{\theta_{2}}\right) \geq\left(\frac{1}{p^{+}}-\frac{1}{\theta_{1}}\right) \\
\quad \times \int_{\Omega}\left|\nabla u_{n}\right|^{p(x)} d x+\left(\frac{1}{q^{+}}-\frac{1}{\theta_{2}}\right) \int_{\Omega}\left|\nabla v_{n}\right|^{q(x)} d x \\
\quad+\left(\frac{1}{\theta_{1}}-\frac{1}{\alpha^{-}}\right) \int_{\Omega}\left|u_{n}\right|^{\alpha(x)} d x+\left(\frac{1}{\theta_{2}}-\frac{1}{\beta^{-}}\right) \int_{\Omega}\left|v_{n}\right|^{\beta(x)} d x \\
\quad+\lambda \int_{\Omega}\left[\frac{u_{n}}{\theta_{1}} \frac{\partial F}{\partial u}\left(x, u_{n}, v_{n}\right)\left(x, u_{n}, v_{n}\right)+\frac{v_{n}}{\theta_{2}} \frac{\partial F}{\partial v}-F\left(x, u_{n}, v_{n}\right)\right] d x .
\end{aligned}
$$

Using $\left(\mathcal{H}_{3}\right)$, we get

$$
\begin{aligned}
& I_{\lambda}\left(u_{n}, v_{n}\right)-I_{\lambda}^{\prime}\left(u_{n}, v_{n}\right)\left(\frac{u_{n}}{\theta_{1}}, \frac{v_{n}}{\theta_{2}}\right) \\
& \geq\left(\frac{1}{p^{+}}-\frac{1}{\theta_{1}}\right) \int_{\Omega}\left|\nabla u_{n}\right|^{p(x)} d x+\left(\frac{1}{q^{+}}-\frac{1}{\theta_{2}}\right) \int_{\Omega}\left|\nabla v_{n}\right|^{q(x)} d x .
\end{aligned}
$$

Proposition 2 gives us

$$
\begin{aligned}
& I_{\lambda}\left(u_{n}, v_{n}\right)-I_{\lambda}^{\prime}\left(u_{n}, v_{n}\right)\left(\frac{u_{n}}{\theta_{1}}, \frac{v_{n}}{\theta_{2}}\right) \geq\left(\frac{1}{p^{+}}-\frac{1}{\theta_{1}}\right) \min \left(\left\|u_{n}\right\|_{1, p(x)}^{p^{+}},\left\|u_{n}\right\|_{1, p(x)}^{p^{-}}\right) \\
& +\left(\frac{1}{q^{+}}-\frac{1}{\theta_{2}}\right) \min \left(\left\|v_{n}\right\|_{1, q(x)}^{q^{+}},\left\|v_{n}\right\|_{1, q(x)}^{q-}\right),
\end{aligned}
$$

thus

$$
\begin{aligned}
C+o(1) \geq & \left(\frac{1}{p^{+}}-\frac{1}{\theta_{1}}\right) \min \left(\left\|u_{n}\right\|_{1, p(x)}^{p^{+}},\left\|u_{n}\right\|_{1, p(x)}^{p^{-}}\right) \\
& +\left(\frac{1}{q^{+}}-\frac{1}{\theta_{2}}\right) \min \left(\left\|v_{n}\right\|_{1, q(x)}^{q^{+}},\left\|v_{n}\right\|_{1, q(x)}^{q-}\right) .
\end{aligned}
$$

Now, without loss of generality, we have may $\left\|u_{n}\right\|_{1, p(x)} \geq\left\|v_{n}\right\|_{1, q(x)}$. Therefore, for $n$ large enough, we get

$$
\begin{aligned}
C+o(1) \geq & \left(\frac{1}{p^{+}}-\frac{1}{\theta_{1}}\right) \min \left(\left\|v_{n}\right\|_{1, q(x)}^{p^{+}},\left\|v_{n}\right\|_{1, q(x)}^{p-}\right) \\
& +\left(\frac{1}{q^{+}}-\frac{1}{\theta_{2}}\right) \min \left(\left\|v_{n}\right\|_{1, q(x)}^{q^{+}},\left\|v_{n}\right\|_{1, q(x)}^{q-}\right)
\end{aligned}
$$

hence, $\left(u_{n}, v_{n}\right)$ is bounded in $X$.

Lemma 3. Let $\left(u_{n}, v_{n}\right)_{n \in \mathbb{N}} \subset X$ be a Palais-Smale sequence with energy level $C$, if

$$
C<\inf \left(\left(\frac{1}{p^{+}}-\frac{1}{\alpha^{-}}\right) S_{p}^{N},\left(\frac{1}{q^{+}}-\frac{1}{\beta^{-}}\right) S_{q}^{N}\right),
$$

then there exists a subsequence strongly convergent in $X . S_{p}$ and $S_{q}$ are the best Sobolev constants corresponding to the embedding $W^{1, p(x)}(\Omega) \hookrightarrow L^{\alpha(x)}(\Omega)$, $W^{1, q(x)}(\Omega) \hookrightarrow L^{\beta(x)}(\Omega)$ respectively. 
Proof. By Lemma $2\left(u_{n}, v_{n}\right)_{n \in \mathbb{N}}$ is bounded in $X$, passing to a subsequence, still denoted by $\left(u_{n}, v_{n}\right)_{n \in \mathbb{N}}$ weakly convergent in $X$, then there exists positive and bounded measures $\mu, \nu, \widetilde{\mu}, \widetilde{\nu}$ in $\Omega$ such that

$$
\left|\nabla u_{n}\right|^{p(x)} \rightarrow \mu, \quad\left|u_{n}\right|^{\alpha(x)} \rightarrow \nu,
$$

$\left(\right.$ respectively $\left.\left|\nabla v_{n}\right|^{q(x)} \rightarrow \widetilde{\mu},\left|v_{n}\right|^{\beta(x)} \rightarrow \widetilde{\nu}\right)$. Hence by Proposition 8 , if $J=\emptyset$ then $u_{n} \rightarrow u$ in $L^{\alpha(x)}(\Omega)$. Let us show that if

$$
C<\inf \left(\left(\frac{1}{p^{+}}-\frac{1}{\alpha^{-}}\right) S_{p}^{N},\left(\frac{1}{q^{+}}-\frac{1}{\beta^{-}}\right) S_{q}^{N}\right)
$$

and $\left(u_{n}, v_{n}\right)_{n \in \mathbb{N}}$ is a Palais-Smale sequence with energy level $C$ then $J=\emptyset$ (respectively $\bar{J}=\emptyset$ ). Suppose that $J \neq \emptyset$, for any $j \in J$, let $\phi_{j, \varepsilon} \in C_{0}^{\infty}\left(B_{2 \varepsilon}\left(x_{j}\right)\right.$ ) such that $0 \leq \phi<1,\left|\nabla \phi_{j, \varepsilon}\right| \leq C / \varepsilon$ and $\phi_{j, \varepsilon} \equiv 1$ on $B\left(x_{j}, \varepsilon\right)$. Substitute $\varphi$ for $\phi_{j, \varepsilon} u_{n}$ and $\psi$ for 0 in $I_{\lambda}^{\prime}\left(u_{n}, v_{n}\right)(\varphi, \psi)$ and using the fact that $I_{\lambda}^{\prime}\left(u_{n}, v_{n}\right) \rightarrow 0$ in $X^{*}$, we obtain

$$
\begin{aligned}
\lim _{n \rightarrow \infty} & \int_{\Omega}\left|\nabla u_{n}\right|^{p(x)-2} \nabla u_{n} \nabla \phi_{j, \varepsilon} u_{n} d x=\lim _{n \rightarrow \infty}\left[\int_{\Omega}-\left|\nabla u_{n}\right|^{p(x)} \phi_{j, \varepsilon} d x\right. \\
& \left.+\int_{\Omega}\left|u_{n}\right|^{\alpha(x)} \phi_{j, \varepsilon} d x+\lambda \int_{\Omega} \frac{\partial F}{\partial u}\left(x, u_{n}, v_{n}\right) \phi_{j, \varepsilon} u_{n} d x\right] .
\end{aligned}
$$

Because of the compactness of $\frac{\partial F}{\partial u}$ and Proposition 8 we get

$$
\begin{aligned}
\lim _{n \rightarrow \infty} & \int_{\Omega}\left|\nabla u_{n}\right|^{p(x)-2} \nabla u_{n} \nabla \phi_{j, \varepsilon} u_{n} d x \\
& =\int_{\Omega}-\phi_{j, \varepsilon} d \mu+\lambda \lim _{n \rightarrow \infty} \int_{\Omega} \frac{\partial F}{\partial u}\left(x, u_{n}, v_{n}\right) \phi_{j, \varepsilon} u_{n} d x+\int_{\Omega} \phi_{j, \varepsilon} d \nu .
\end{aligned}
$$

By Hölder's inequality, it is easy to check that

$$
\lim _{\varepsilon \rightarrow 0} \int_{\Omega}\left|\nabla u_{n}\right|^{p(x)-2} \nabla u_{n} \nabla \phi_{j, \varepsilon} u_{n} d x=0, \text { as } n \rightarrow+\infty \text {. }
$$

Indeed,

$$
\begin{aligned}
& \left.\left.\lim _{n \rightarrow \infty}\left|\int_{\Omega}\right| \nabla u_{n}\right|^{p(x)-2} \nabla u_{n} \nabla \phi_{j, \varepsilon} u_{n} d x\left|\leq \limsup _{n \rightarrow 0} \int_{\Omega}\right| \nabla u_{n}\right|^{p(x)-1} \\
& \quad \times\left|\nabla \phi_{j, \varepsilon} u_{n}\right| d x \leq\left.\left. 2 \limsup _{n \rightarrow 0}|| \nabla u_{n}\right|^{p(x)-1}\right|_{p^{\prime}(x)}\left|\nabla \phi_{j, \varepsilon} u_{n}\right|_{p(x)} .
\end{aligned}
$$

Since $\left(u_{n}\right)$ is bounded, the real-valued sequence $\left.\left.|| \nabla u_{n}\right|^{p(x)-1}\right|_{p^{\prime}(x)}$ is also bounded. In view of Proposition 2, it suffices to write

$$
\left|\nabla u_{n}\right|_{p^{\prime}(x)}^{(p(x)-1) p^{\prime i}} \leq\left(\left|\nabla u_{n}\right|^{p(x)-1}\right)=\int_{\Omega}\left|\nabla u_{n}\right|^{p(x)} d x \leq\left|\nabla u_{n}\right|_{p(x)}^{p^{j}}, i, j=+ \text { or }-.
$$


Moreover $\left(u_{n}\right)$ is bounded in $W^{1, p(x)}\left(B_{2 \varepsilon}\left(x_{j}\right)\right)$, then there exists a subsequence denoted again $\left(u_{n}\right)$ weakly convergente to $u$ in $L^{p(x)}\left(B_{2 \varepsilon}\left(x_{j}\right)\right)$. Hence

$$
\begin{aligned}
\lim _{n \rightarrow \infty} & \left.\left.\left|\int_{\Omega}\right| \nabla u_{n}\right|^{p(x)-2} \nabla u_{n} \nabla \phi_{j, \varepsilon} u_{n} d x|\leq c| \nabla \phi_{j, \varepsilon} u\right|_{p(x)} \\
& \leq\left.\left.\left.\left. 2 c \limsup _{\varepsilon \rightarrow 0}|| \nabla \phi_{j, \varepsilon}\right|^{p(x)}\right|_{\left(\frac{p^{*}(x)}{p(x)}\right)^{\prime}, B_{2 \varepsilon}\left(x_{j}\right)}|| u\right|^{p(x)}\right|_{\frac{p^{*}(x)}{p(x)}, B_{2 \varepsilon}\left(x_{j}\right)} \\
& =\left.\left.\left.\left.2 c \limsup _{\varepsilon \rightarrow 0}|| \nabla \phi_{j, \varepsilon}\right|^{p(x)}\right|_{\frac{N}{p(x)}, B_{2 \varepsilon}\left(x_{j}\right)}|| u\right|^{p(x)}\right|_{\frac{N}{N-p(x)}, B_{2 \varepsilon}\left(x_{j}\right)}
\end{aligned}
$$

and

$$
\begin{aligned}
\int_{B_{2 \varepsilon}\left(x_{j}\right)}\left(\left|\nabla \phi_{j, \varepsilon}\right|^{p(x)}\right)^{\left(\frac{p^{*}(x)}{p(x)}\right)^{\prime}} d x & =\int_{B_{2 \varepsilon}\left(x_{j}\right)}\left|\nabla \phi_{j, \varepsilon}\right|^{N} d x \\
& \leq(C / \varepsilon)^{N} \operatorname{meas}\left(B_{2 \varepsilon}\left(x_{j}\right)\right)=\left(4^{N} / N\right) \omega_{N},
\end{aligned}
$$

where $\omega_{N}$ is the surface area of an $N$-dimensional unit sphere. Since $\int_{B_{2 \varepsilon}\left(x_{j}\right)}\left(|u|^{p(x)}\right)^{\frac{p^{*}(x)}{p(x)}} d x \rightarrow 0$, as $\varepsilon \rightarrow 0$, we obtain $\left|\nabla \phi_{j, \varepsilon} u\right|_{p(x)} \rightarrow 0$, which implies

$$
\lim _{\varepsilon \rightarrow 0} \int_{\Omega}\left|\nabla u_{n}\right|^{p(x)-2} \nabla u_{n} \nabla \phi_{j, \varepsilon} u_{n} d x=0, \text { as } n \rightarrow+\infty .
$$

Similarly, we can also get

$$
\lim _{\varepsilon \rightarrow 0} \int_{\Omega} \frac{\partial F}{\partial u}\left(x, u_{n}, v_{n}\right) \phi_{j, \varepsilon} u_{n} d x=0, \text { as } n \rightarrow+\infty
$$

Indeed, using Hölder's inequality with $\left(\mathcal{H}_{2}\right)$ and since $0 \leq \phi_{j, \varepsilon} \leq 1$ we obtain

$$
\begin{aligned}
& \lim _{n \rightarrow 0} \int_{\Omega} \frac{\partial F}{\partial u}\left(x, u_{n}, v_{n}\right) \phi_{j, \varepsilon} u_{n} d x \leq \lim _{n \rightarrow 0} \int_{\Omega}\left(a_{1}(x)\left|u_{n}\right|^{p_{1}^{-}-1}\right. \\
& \left.\quad+a_{2}(x)\left|v_{n}\right|^{p_{1}^{+}-1}\right) \phi_{j, \varepsilon} u_{n} d x \leq \lim _{n \rightarrow 0}\left(\left.\left.\left|a_{1}(x)\right|_{\alpha_{1}}|| u_{n}\right|^{p_{1}^{-}-1}\right|_{p^{*}}\left|\phi_{j, \varepsilon} u_{n}\right|_{p}\right. \\
& \left.\quad+\left.\left.\left|a_{2}(x)\right|_{\beta}|| v_{n}\right|^{p_{1}^{+}-1}\right|_{q^{*}}\left|\phi_{j, \varepsilon} u_{n}\right|_{p^{*}}\right) \leq \lim _{n \rightarrow 0}\left(\left.\left.\left|a_{1}(x)\right|_{\alpha_{1}}|| u_{n}\right|^{p_{1}^{-}-1}\right|_{p^{*}}\left|u_{n}\right|_{p, B_{2 \varepsilon}}\right. \\
& \left.\quad+\left.\left.\left|a_{2}(x)\right|_{\beta}|| v_{n}\right|^{p_{1}^{+}-1}\right|_{q^{*}}\left|u_{n}\right|_{p^{*}, B_{2 \varepsilon}}\right) .
\end{aligned}
$$

The above propositions yield

$$
\begin{aligned}
& \lim _{n \rightarrow 0} \int_{\Omega} \frac{\partial F}{\partial u}\left(x, u_{n}, v_{n}\right) \phi_{j, \varepsilon} u_{n} d x \leq \lim _{n \rightarrow 0}\left(\left|a_{1}\right|_{\alpha_{1}}|| u_{n}||_{p, \Omega}^{p_{1}^{-}-1}\left\|u_{n}\right\|_{p ; B_{2 \varepsilon}}\right. \\
& \left.\quad+\left|a_{2}\right|{ }_{\beta}|| v_{n}||_{q, \Omega}^{p_{1}^{+}-1}|| u_{n} \|_{p ; B_{2 \varepsilon}}\right) \\
& \quad \leq \lim _{n \rightarrow 0}\left(\left|a_{1}\right|_{\alpha_{1}}|| u_{n}||_{p, \Omega}^{p_{1}^{-}-1}+\left|a_{2}\right|_{\beta}|| v_{n}||_{q, \Omega}^{p_{1}^{+}-1}\right)\left\|u_{n}\right\|_{p ; B_{2 \varepsilon}}
\end{aligned}
$$

and this last goes to zero because of

$$
\left|a_{1}\right|_{\alpha_{1}}|| u_{n}||_{p, \Omega}^{p_{1}^{-}-1}+\left|a_{2}\right|_{\beta}|| v_{n}||_{q, \Omega}^{p_{1}^{+}-1}<+\infty .
$$


Thus, it follows from (3.1) that $\mu_{j}=\nu_{j}$ for any $j \in J$. Using again Proposition 8 , we conclude that $\nu_{j} \geq S_{p}^{N}$. Consequently

$$
\int_{\Omega}\left|u_{n}\right|^{\alpha(x)} d x \rightarrow \int_{\Omega} d \nu \geq \int_{\Omega}|u|^{\alpha(x)} d x+S_{p}^{N} \sum_{j \in J} \delta_{x_{j}} \geq \int_{\Omega}|u|^{\alpha(x)} d x+S_{p}^{N} \text { Card } J .
$$

If Card $J=\infty$, we get a contradiction.

On the other hand, we have

$$
\begin{aligned}
I_{\lambda} & \left(u_{n}, v_{n}\right)-I_{\lambda}^{\prime}\left(u_{n}, v_{n}\right)\left(\frac{u_{n}}{p^{+}}, \frac{v_{n}}{q^{+}}\right)=\int_{\Omega} \frac{1}{p(x)}\left|\nabla u_{n}\right|^{p(x)} d x+\int_{\Omega} \frac{1}{q(x)}\left|\nabla v_{n}\right|^{q(x)} d x \\
& -\int_{\Omega} \frac{1}{\alpha(x)}\left|u_{n}\right|^{\alpha(x)} d x-\int_{\Omega} \frac{1}{\beta(x)}\left|v_{n}\right|^{\beta(x)} d x-\int_{\Omega} \lambda F\left(x, u_{n}, v_{n}\right) d x \\
& -\frac{1}{p^{+}} \int_{\Omega}\left|\nabla u_{n}\right|^{p(x)} d x-\frac{1}{q^{+}} \int_{\Omega}\left|\nabla v_{n}\right|^{q(x)} d x+\frac{1}{p^{+}} \int_{\Omega}\left|u_{n}\right|^{\alpha(x)} d x \\
& +\frac{1}{q^{+}} \int_{\Omega}\left|v_{n}\right|^{\beta(x)} d x+\frac{\lambda}{p^{+}} \int_{\Omega} \frac{\partial F}{\partial u}\left(x, u_{n}, v_{n}\right) u_{n} d x+\frac{\lambda}{q^{+}} \int_{\Omega} \frac{\partial F}{\partial v}\left(x, u_{n}, v_{n}\right) v_{n} d x \\
\geq & \frac{1}{p^{+}} \int_{\Omega}\left|\nabla u_{n}\right|^{p(x)} d x+\frac{1}{q^{+}} \int_{\Omega}\left|\nabla v_{n}\right|^{q(x)} d x-\frac{1}{\alpha^{-}} \int_{\Omega}\left|u_{n}\right|^{\alpha(x)} d x \\
- & \int_{\Omega} \frac{1}{\beta^{-}}\left|v_{n}\right|^{\beta(x)} d x-\int_{\Omega} \lambda F\left(x, u_{n}, v_{n}\right) d x-\frac{1}{p^{+}} \int_{\Omega}\left|\nabla u_{n}\right|^{p(x)} d x \\
- & \frac{1}{q^{+}} \int_{\Omega}\left|\nabla v_{n}\right|^{q(x)} d x+\frac{1}{p^{+}} \int_{\Omega}\left|u_{n}\right|^{\alpha(x)} d x+\frac{1}{q^{+}} \int_{\Omega}\left|v_{n}\right|^{\beta(x)} d x \\
+ & \frac{\lambda}{p^{+}} \int_{\Omega} \frac{\partial F}{\partial u}\left(x, u_{n}, v_{n}\right) u_{n} d x+\frac{\lambda}{q^{+}} \int_{\Omega} \frac{\partial F}{\partial v}\left(x, u_{n}, v_{n}\right) v_{n} d x
\end{aligned}
$$

hence

$$
C \geq\left(\frac{1}{p^{+}}-\frac{1}{\alpha^{-}}\right) \int_{\Omega}\left|u_{n}\right|^{\alpha(x)} d x+\left(\frac{1}{q^{+}}-\frac{1}{\beta^{-}}\right) \int_{\Omega}\left|v_{n}\right|^{\beta(x)} d x .
$$

When $n \rightarrow \infty$ we obtain

$$
C \geq\left(\frac{1}{p^{+}}-\frac{1}{\alpha^{-}}\right)\left(\int_{\Omega}|u|^{\alpha(x)} d x+\sum_{j \in J} \nu_{j} \delta_{x_{j}}\right)+\left(\frac{1}{q^{+}}-\frac{1}{\beta^{-}}\right)\left(\int_{\Omega}|v|^{\beta(x)} d x+\sum_{j \in \bar{J}} \bar{\nu}_{j} \delta_{x_{j}}\right),
$$

but, $\nu_{j} \geq S_{p}^{N}\left(\right.$ and $\left.\bar{\nu}_{j} \geq S_{q}^{N}\right)$

$$
C \geq\left(\frac{1}{p^{+}}-\frac{1}{\alpha^{-}}\right)\left(\int_{\Omega}|u|^{\alpha(x)} d x+S_{p}^{N} \operatorname{Card} J\right)+\left(\frac{1}{q^{+}}-\frac{1}{\beta^{-}}\right)\left(\int_{\Omega}|v|^{\beta(x)} d x+S_{q}^{N} \operatorname{Card} \bar{J}\right) .
$$

Suppose that $J \cup \bar{J} \neq \emptyset$ and thus

$$
\begin{aligned}
& C \geq \inf \left(\left(\frac{1}{p^{+}}-\frac{1}{\alpha^{-}}\right) S_{p}^{N},\left(\frac{1}{q^{+}}-\frac{1}{\beta^{-}}\right) S_{q}^{N}\right), \\
& C \geq\left(\frac{1}{p^{+}}-\frac{1}{\alpha^{-}}\right)\left(\int_{\Omega}|u|^{\alpha(x)} d x+S_{p}^{N} \operatorname{Card} J\right)+\left(\frac{1}{q^{+}}-\frac{1}{\beta^{-}}\right)\left(\int_{\Omega}|v|^{\beta(x)} d x+S_{q}^{N} \operatorname{Card} \bar{J}\right),
\end{aligned}
$$




$$
C \geq \inf \left(\left(\frac{1}{p^{+}}-\frac{1}{\alpha^{-}}\right) S_{p}^{N},\left(\frac{1}{q^{+}}-\frac{1}{\beta^{-}}\right) S_{q}^{N}\right) .
$$

Therefore, if $C<\inf \left(\left(\frac{1}{p^{+}}-\frac{1}{\alpha^{-}}\right) S_{p}^{N},\left(\frac{1}{q^{+}}-\frac{1}{\beta^{-}}\right) S_{q}^{N}\right)$, the set $J \cup \bar{J}$ is empty, which means that $\left\|u_{n}\right\|_{\alpha(x)} \rightarrow\|u\|_{\alpha(x)}$ and $\left\|v_{n}\right\|_{\beta(x)} \rightarrow\|v\|_{\beta(x)}$. Taking this together with the fact that $\left(u_{n}, v_{n}\right) \rightarrow(u, v)$ in $X$, we have $u_{n} \rightarrow u$ strongly in $L^{\alpha(x)}(\Omega)$ and $v_{n} \rightarrow v$ strongly in $L^{\beta(x)}(\Omega)$. On the other hand

$$
\begin{aligned}
& \left(J^{\prime}\left(u_{n}, v_{n}\right)-J^{\prime}\left(u_{m}, v_{m}\right)\right)\left(u_{n}-u_{m}, 0\right)=\left(I_{\lambda}^{\prime}\left(u_{n}, v_{n}\right)\right. \\
& \left.\left.\quad-I_{\lambda}^{\prime}\left(u_{m}, v_{m}\right)\right)\left(u_{n}-u_{m}, 0\right)+\lambda\left(K^{\prime}\left(u_{n}, v_{n}\right)-K^{\prime}\left(u_{m}, v_{m}\right)\right)\left(u_{n}-u_{m}, 0\right)\right) \\
& \left.\quad+\left(L^{\prime}\left(u_{n}, v_{n}\right)-L^{\prime}\left(u_{m}, v_{m}\right)\right)\left(u_{n}-u_{m}, 0\right)\right)
\end{aligned}
$$

thus $I^{\prime}\left(u_{n}, v_{n}\right) \rightarrow 0$, i.e $I^{\prime}\left(u_{n}, v_{n}\right)$ is a Cauchy sequence in $X^{*}$. Moreover, by Hölder's inequality

$$
\begin{aligned}
& \left(L^{\prime}\left(u_{n}, v_{n}\right)-L^{\prime}\left(u_{m}, v_{m}\right)\left(u_{n}-u_{m}, 0\right)\right) \\
& \quad=\int\left(\left|u_{n}\right|^{\alpha(x)-2} u_{n}-\left|u_{m}\right|^{\alpha(x)-2} u_{m}\right)\left(u_{n}-u_{m}\right) d x \\
& \quad \leq\left\|\left|u_{n}\right|^{\alpha(x)-2} u_{n}-\left|u_{m}\right|^{\alpha(x)-2} u_{m}\right\|_{L^{\frac{\alpha(x)}{\alpha(x)-1}}(\Omega)}\left\|u_{n}-u_{m}\right\|_{L^{\alpha(x)}(\Omega)} .
\end{aligned}
$$

Since $\left(u_{n}\right)$ is a Cauchy sequence in $L^{\alpha(x)}(\Omega), L^{\prime}\left(u_{n}, v_{n}\right)$ is a Cauchy sequence in $X^{*}$. The compactness of $K^{\prime}$ gives

$$
\left(u_{n}, v_{n}\right) \rightarrow(u, v) \Rightarrow K^{\prime}\left(u_{n}, v_{n}\right) \rightarrow K^{\prime}(u, v),
$$

i.e. $K^{\prime}\left(u_{n}, v_{n}\right)$ is a Cauchy sequence in $X^{*}$.

Therefore, according to the elementary inequalities

$$
\begin{aligned}
& |\lambda-\mu|^{p(x)} \\
& \quad \leq\left\{\begin{array}{l}
\left(|\lambda|^{p(x)-2} \lambda-|\mu|^{p(x)-2} \mu\right) \cdot(\lambda-\mu), \text { if } p(x) \geq 2, \\
{\left[\left(|\lambda|^{p(x)-2} \lambda-|\mu|^{p(x)-2} \mu\right) \cdot(\lambda-\mu)\right]^{\frac{p(x)}{2}}(|\lambda|-|\mu|)^{\frac{p(x)(2-p(x))}{2}},} \\
\text { if } 1<p(x)<2,
\end{array}\right.
\end{aligned}
$$

$\forall \lambda, \mu \in \mathbb{R}^{N}$, where . denote the standard inner product in $\mathbb{R}^{N}$. Replacing $\lambda$ and $\mu$ by $\nabla u_{n}$ and $\nabla u_{m}$ respectively and integrating over $\Omega$, we obtain

$$
\left\|u_{n}-u_{m}\right\|^{p^{-}} \leq\left|\left(J^{\prime}\left(u_{n}, v_{n}\right)-J^{\prime}\left(u_{m}, v_{m}\right)\right)\left(u_{n}-u_{m}, 0\right)\right|, \quad \text { if } p(x) \geq 2,
$$

and if $1<p(x)<2$, we get

$$
\begin{aligned}
\left\|u_{n}-u_{m}\right\|^{2} \leq \mid & \left(J^{\prime}\left(u_{n}, v_{n}\right)-J^{\prime}\left(u_{m}, v_{m}\right)\right)\left(u_{n}-u_{m}, 0\right) \mid \\
& \times\left(\left\|u_{n}\right\|_{1, p(x)}^{p^{-}}-\left\|u_{m}\right\|_{1, p(x)}^{p^{-}}\right)^{\frac{p^{-}\left(2-p^{+}\right)}{2}} .
\end{aligned}
$$

Taking into account the fact that $\left(u_{n}\right)$ is bounded in $W^{1, p(x)}(\Omega)$

$$
\left(J^{\prime}\left(u_{n}, v_{n}\right)-J^{\prime}\left(u_{m}, v_{m}\right)\right)\left(u_{n}-u_{m}, 0\right) \rightarrow 0, \text { as } n, m \rightarrow \infty,
$$


we find that $\left(u_{n}\right)$ is a Cauchy sequence in $W^{1, p(x)}(\Omega)$. We proceed similarly for $\left(v_{n}\right)$ with $\left(J^{\prime}\left(u_{n}, v_{n}\right)-J^{\prime}\left(u_{m}, v_{m}\right)\right)\left(0, v_{n}-v_{m}\right)$.

Now we are in position to prove Theorem 1.

Proof. The proof is an immediate consequence of the mountain pass theorem, Lemma 2 and Lemma 3. Precisely, it suffices to verify that $I_{\lambda}$ has the mountainpass geometry and that $I_{\lambda}(t u, t v)<0$ for some $t>0$. About the later condition, we have

$$
\begin{aligned}
I_{\lambda}(u, v)= & \int_{\Omega} \frac{1}{p(x)}|\nabla u|^{p(x)} d x+\int_{\Omega} \frac{1}{q(x)}|\nabla v|^{q(x)} d x-\int_{\Omega} \frac{1}{\alpha(x)}|u|^{\alpha(x)} d x \\
& +\int_{\Omega} \frac{1}{\beta(x)}|v|^{\beta(x)} d x-\lambda \int_{\Omega} F(x, u(x), v(x)) d x .
\end{aligned}
$$

Then, because of $\int_{\Omega} F(x, u(x), v(x)) d x>0$, it's clear that for $(\omega, \widetilde{\omega}) \in X /\{(0,0)\}$ and any $t>1$

$$
\begin{aligned}
I_{\lambda}(t \omega, t \widetilde{\omega}) \leq & t^{p^{+}} \int_{\Omega} \frac{1}{p(x)}|\nabla \omega|^{p(x)} d x+t^{q^{+}} \int_{\Omega} \frac{1}{q(x)}|\nabla \widetilde{\omega}|^{q(x)} d x \\
& -t^{\alpha^{-}} \int_{\Omega} \frac{1}{\alpha(x)}|\omega|^{\alpha(x)} d x-t^{\beta^{-}} \int_{\Omega} \frac{1}{\beta(x)}|\widetilde{\omega}|^{\beta(x)} d x,
\end{aligned}
$$

which tends to $-\infty$ as $t \rightarrow+\infty$ since $\alpha^{-}>p^{+}$and $\beta^{-}>q^{+}$.

On the other hand, for $\|(u, v)\|=R$ is small enough and from $\left(\mathcal{H}_{4}\right)$, we get

$$
\begin{aligned}
I_{\lambda}(u, v) \geq \frac{1}{p^{+}}|\nabla u|_{L^{p(x)}}^{p^{+}}+\frac{1}{q^{+}}|\nabla v|_{L^{q(x)}}^{q^{+}}-\frac{1}{\alpha^{-}}|u|_{L^{\alpha(x)}}^{\alpha^{-}}-\frac{1}{\beta^{-}}|v|_{L^{\beta(x)}}^{\beta^{-}} & \\
& -\lambda|u|_{L_{1}^{r_{1}(x)}}^{r^{-}}-\lambda|v|_{L_{2}^{r_{2}(x)}}^{r^{-}} \\
\geq & \frac{1}{p^{+}}\|u\|_{1, p(x)}^{p^{+}}+\frac{1}{q^{+}}\|v\|_{1, q(x)}^{q^{+}}-\frac{c}{\alpha^{-}}\|u\|_{1, p(x)}^{\alpha^{-}}-\frac{c}{\beta^{-}}\|v\|_{1, q(x)}^{\beta^{-}} \\
& -\lambda c\|u\|_{1, p(x)}^{r_{1}^{-}}-\lambda c\|v\|_{1, p(x)}^{r_{2}^{-}} \\
\geq & \left(\frac{1}{p^{+}}\|u\|_{1, p(x)}^{p^{+}}-\frac{c}{\alpha^{-}}\|u\|_{1, p(x)}^{\alpha^{-}}-\lambda c\|u\|_{1, p(x)}^{r_{1}^{-}}\right) \\
& +\left(\frac{1}{q^{+}}\|v\|_{1, q(x)}^{q^{+}}-\frac{c}{\beta^{-}}\|v\|_{1, q(x)}^{\beta^{-}}-\lambda c\|v\|_{1, q(x)}^{r_{2}^{-}}\right) .
\end{aligned}
$$

So, it is easy to check that $g_{i}(R)>a>0, i=1,2$, where

$$
\left\{\begin{array}{l}
g_{1}(t)=\frac{1}{p^{+}} t^{p^{+}}-\frac{c}{\alpha^{-}} t^{\alpha^{-}}-\lambda c t^{r_{1}^{-}} \\
g_{2}(t)=\frac{1}{q^{+}} t^{q^{+}}-\frac{c}{\beta^{-}} t^{\beta^{-}}-\lambda c t^{r_{2}^{-}}
\end{array}\right.
$$

since $r_{1}^{-}, \alpha^{-}>p^{+}$and $r_{2}^{-}, \beta^{-}>q^{+}$. That means the existence of an element $\left(u_{0}, v_{0}\right)$ of $X$ such that $I_{\lambda}\left(u_{0}, v_{0}\right)<0$. Consequentely, the critical value is

$$
C:=\inf _{\xi \in \Gamma} \sup _{t \in[0,1]} I_{\lambda}(\xi(t)),
$$


where

$$
\Gamma=\left\{\xi:[0,1] \rightarrow X ; \text { continuous and } \xi(0)=(0,0), \xi(1)=\left(u_{0}, v_{0}\right)\right\} .
$$

That concludes the proof.

\section{References}

[1] P. Amster, P. De Nápoli and M.C. Mariani. Existence of solutions for elliptic systems with critical Sobolev exponent. EJDE, 2002(49):1-13, 2002.

[2] L. Boccardo and D.G. de Figueiredo. Some remarks on a system of quasilinear elliptic equations. Nonlinear Differen. Equat. and Appl, 9:309-323, 2002.

[3] J.F. Bonder, N. Saintier and A. Silva. Existence of solution to a critical equation with variable exponent. Ann. Acad. Sci. Fenn. Math., 37:579-594, 2012.

[4] J.F. Bonder, N. Saintier and A. Silva. On the Sobolev embedding theorem for variable exponent spaces in the critical range. JDE, 253(5):1604-1620, 2012.

[5] J.F. Bonder and A. Silva. Concentration-compactness principle for variable exponent spaces and applications. EJDE, 141:1-18, 2010.

[6] J.F. Bonder, S. Martínez and J.D. Rossi. Existence results for gradient elliptic systems with nonlinear boundary conditions. Nonlinear Differ. Equ. Appl., 14(12):153-179, 2007.

[7] H. Brezis and L. Nirenberg. Positive solutions of nonlinear elliptic equations involving critical Sobolev exponents. Commun. Pure. Appl. Math, 36:437-477, 1983.

[8] Y. Chen, S. Levine and M. Rao. Variable exponent, linear growth functionals in image restoration. SIAMJ. Appl. Math., 66(4):1383-1406, 2006.

[9] L. Diening, P. Harjulehto, P. Hästö and M. Rùz̃ička. Lebesgue and Sobolev spaces with variable exponents. Springer, 2010.

[10] L. Diening, P. Hästö and A. Nekvinda. Open problems in variable exponent Lebesgue and Sobolev spaces. FSDONA04 Proceedings, Milovy, Czech Republic, 66:38-58, 2004.

[11] A. Djellit and S. Tas. Quasilinear elliptic systems with critical Sobolev exponents in $\mathbb{R}^{\mathbb{N}}$. Nonlinear Anal, 66(7):1485-1497, 2007.

[12] A. Djellit, Z. Youbi and S. Tas. Existence of solutions for elliptic systems in $\mathbb{R}^{\mathbb{N}}$ involving the $p(x)$-Laplacian. EJDE, 2012(131):1-10, 2012.

[13] D.E. Edmunds and J. Răkosnik. Sobolev embeddings with variable exponent. Studia Math., 143(3):267-293, 2000.

[14] X. L. Fan, Q. H. Zhang and D. Zhao. Eigenvalues of the $p(x)$-Laplacian Dirichlet problem. J. Math. Annl. App, 306(2):306-317, 2005.

[15] X.L. Fan, J. Shen and D. Zhao. Sobolev embedding theorems for spaces $W^{k, p(x)}(\Omega)$. J. Math. Anal. App., 262:749-760, 2001.

[16] X.L. Fan and Q.H. Zhang. Existence of solutions for $p(x)$-Laplacian Dirichlet problem. Nonlinear. Anal, 52:1843-1852, 2003.

[17] X.L. Fan and D. Zhao. On the spaces $L^{p(x)}$ and $W^{m, p(x)}$. J. Math. Anal. Appl, 263:424-446, 2001. 
[18] Y. Fu. The principle of concentration compactness in $L^{p(x)}$ spaces and its application. Nonlinear. Anal, 71(5-6):1876-1892, 2009.

[19] A. El Hamidi. Existence results to elliptic systems with nonstandard growth conditions. J. Math. Anal. App, 300:30-42, 2004.

[20] P. Hästö. The $p(x)$-Laplacian and applications. The Journal of Analysis, 15:53$62,2007$.

[21] H. Hudzik. On generalized Orlicz-Sobolev spaces. Funct. Approximatio Commentarii Mathematici, 4:37-51, 1976.

[22] Y. Jebri. The Mountain pass theorem. Combridge university press, New York, 2003.

[23] O. Kavian. Introduction à la théorie des points critiques et applications aux problèmes elliptiques. Springer-Verlag France, Paris, 1993.

[24] F. Li, Z. Li and L. Pi. Variable exponent functionals in image restoration. App. Math. Comput., 216(3):870-882, 2010.

[25] P.L. Lions. The concentration-compactness principle in calculus of variation, the limit case part 1 and 2. Rev, Mat, Ibroamericana, 1(1):145-201, 1985.

[26] S. Ogras, R.A. Mashiyev, M. Avci and Z. Yucedag. Existence of solution for a class of elliptic systems in $\mathbb{R}^{\mathbb{N}}$ involving the $(p(x), q(x)$ )-Laplacian. J. Inequal. Appl., 612936:1-16, 2008.

[27] S. Samko. On a progress in the theory of Lebesgue spaces with variable exponent: maximal and singular operators. Integral Transforms Spec. Funct., 16(5):461482, 2005.

[28] A. Silva. Multiple solutions for the $p(x)$-Laplace operator with critical growth. Adv. Nonlinear Stud., 11:63-75, 2011.

[29] M. Rùz̃ička. Electrorheological Fluids: Modeling and Mathematical theory. Springer-Verlage, Berlin, 2000.

[30] X. Xu and Y. An. Existence and multiplicity of solutions for elliptic systems with nonstandard growth conditions in $\mathbb{R}^{\mathbb{N}}$. Nonlinear. Anal, 68:956-968, 2008.

[31] Q. Zhang, Y. Guo and G. Chen. Existence and multiple solutions for a variable exponent system. Nonlinear Anal., 73(12):3788-3804, 2010.

[32] X. Zhang and Y. Fu. Solutions of $p(x)$-Laplacian equations with critical exponent and perturbations in $\mathbb{R}^{\mathbb{N}}$. EJDE, 2012(120):1-14, 2012. 